\title{
Application of BP Neural Network in Experimental Teaching Evaluation
}

\author{
Yangming He \\ School of Computer, \\ Jiangxi University of Traditional Chinese Medicine, \\ China
}

\author{
Guohua Jin \\ School of Computer, \\ Jiangxi University of Traditional Chinese Medicine,
}

\begin{abstract}
In this paper, BP neural network is used to evaluate the experimental teaching. This method is different from traditional evaluation. It merges together many features of students' performance in experiments. It can evaluate students all-aroundly. This paper describes how to establish the BP neural network. In the end, practical example is used to prove the validity of the method.
\end{abstract}

Keywords—neural network; experimental teaching; evaluation; students' performance

\section{INTRODUCTION}

In today's undergraduate education process, many courses are demanded to carry out a lot of experimental operations, and most of these courses are basic courses. Experimental skills can not only confirm theoretical knowledge, but also deepen understanding. Evaluating students' performance in experiments has become a key issue. Correct evaluation will become a driving force for students to learn. Many scholars have studied this problem and put forward many methods. These methods are of great guiding significance to experimental teaching in theory.

The evaluation of students' experimental ability is an important part of the experiment. Some parameters reflecting students' abilities are adopted in evaluation, for example, students' experimental design, enthusiasm, experimental results and experimental report quality. The traditional evaluation methods often use the weighted method to calculate the students' score. The fatal drawback of this method is to isolate the parameters that reflect students' abilities in experiment.[1][2]

This paper abandons the simple weighting method in traditional evaluation methods. A powerful mathematical modeling function of BP neural network is used in this paper to get the evaluation results of students' experimental ability.

\section{BP NEURAL NETWORK}

Artificial neural network is a kind of non-linear information processing system which mimics human brain structure and functions. It simulates some basic characteristics of human brain by mathematical method. After more than half a century of development, due to a variety of network structure and algorithm system, artificial neural network has gradually developed into a more perfect theory system. BP neural network is the most widely used in the technology, and it has some outstanding advantages which are self-adaption, self- organization, self-learning ability etc. It is these characteristics that make the method solve many practical problems. In order to better generalize the global optimal problem, many scholars put forward a lot of targeted approach, including the following three aspects of improvement: the first is to improve the training speed of the network, the second is to improve training accuracy, the third is to avoid falling into the local Minimal point.[3]

BP neural network model includes input layer, hidden layer and output layer. The theoretical calculation steps of the BP neural network model are as follows:

The first step is to assign each of the connection weights $w_{i j}, v_{j t}$, the thresholds $\theta_{j}$ and $\gamma_{t}$ random values.

The second step is to calculate the input $a_{j}$ with the input samples $x_{k}=\left(x_{1}^{k}, x_{2}^{k}, \ldots, x_{n}^{k}\right)$, the connection weights $w_{i j}$ and the thresholds $\theta_{j}$. The input $a_{j}$ is the cells of the hidden layer. Then the output $b_{j}$ of the hidden layers is calculated with $a_{j}$ by using the transfer function.

$$
\begin{aligned}
& a_{j}=\sum_{i=1}^{n} w_{i j} x_{i}-\theta_{j}(j=1,2, \ldots, p) \\
& b_{j}=f\left(a_{j}\right) \quad(j=1,2, \ldots, p)
\end{aligned}
$$

The third step is to calculate the output $L_{t}$ of each unit of the output layer using the output $b_{j}$, the weight $v_{j}$ and the threshold value $\gamma_{t}$ of the hidden layer. Then the actual output $C_{t}$ of each cell of the output layer is calculated by transfer function.

$$
\begin{aligned}
& L_{t}=\sum_{i=1}^{p} v_{j t} b_{i}-\gamma_{t}(t=1,2, \ldots, q) \\
& C_{t}=f\left(L_{t}\right) \quad(t=1,2, \ldots, q)
\end{aligned}
$$


The forth step is to calculate the unit training error $d_{t}^{k}$ of the output layer by using the network target vector $T_{k}=\left(y_{1}^{k}, y_{2}^{k}, \ldots, y_{q}^{k}\right)$ and the actual output $C_{t}$ of the network

$$
d_{t}^{k}=\left(y_{t}^{k}-C_{t}\right) \bullet C_{t}\left(1-C_{t}\right)(t=1,2, \ldots, q)
$$

The fifth step is to calculate the training error $e_{j}^{k}$ of each unit of the hidden layer using the connection weight $v_{j t}$, the training error $d_{t}$ of the output layer and the output $b_{j}$ of the intermediate layer.

$$
e_{j}^{k}=\left[\sum_{t=1}^{q} d_{t} \bullet v_{j t}\right] \bullet b_{j}\left(1-b_{j}\right)
$$

The sixth step is to adjust the connection weight $v_{j t}$ and threshold $\gamma_{t}$ using the training error $d_{t}^{k}$ of each cell of the output layer and the output $b_{j}$ of the hidden layer unit.

$$
\begin{aligned}
& v_{j t}(N+1)=v_{j t}(N)+\alpha \bullet d_{t}^{k} \bullet b_{j} \\
& \gamma_{t}(N+1)=\gamma_{t}(N)+\alpha \bullet d_{t}^{k} \\
& t=1,2, \ldots, q ; j=1,2, \ldots, p ; 0<\alpha<1
\end{aligned}
$$

The seventh step is to adjust the connection weight $w_{i j}$ and the threshold value $\theta_{j}$ using the training error $e_{j}^{k}$ of the respective layers of the hidden layer and the input $x_{k}$ of the input layer units.

$$
\begin{gathered}
w_{i j}(N+1)=w_{i j}(N)+\beta \bullet e_{j}^{k} \bullet x_{i}^{k} \\
\theta_{j}(N+1)=\theta_{j}(N)+\beta \bullet e_{j}^{k} \\
i=1,2, \ldots, n ; \quad j=1,2, \ldots, p ; \quad 0<\beta<1 .
\end{gathered}
$$

The eighth step is to determine whether network learning meet the accuracy requirements of the network. It can use the energy function $E=\frac{1}{2} \sum_{i=1}^{q}\left(y_{i}-C_{i}\right)$. If the energy function is less than a preset minimum value, then the network convergence and the training meet the accuracy requirements. In contrast, it needs to adjust and select the parameters, or reanalysis the correlation of the input factor and output factor.

\section{Design of the BP Neural Network}

The results show that the three-layer BP neural network with a single hidden layer is sufficient to perform normal function mapping. Therefore, in this paper a 3-layer BP neural network with a hidden layer is used. The BP neural network consists of one input layer, one hidden layer and one output layer.[4]-[9]

It is realized in Matlab. The students studying the principles of computer course participate in the experiment. Five parameters are chosen to be the input of the BP neural network. They are feasibility of experimental scheme, standardization of instrument operation, reliability of experimental data, accuracy of result analysis and experimental attitude.

After selecting the evaluation parameters, we need to determine the network structure. According to Kolmogov theorem, The structure of the network can be $\mathrm{N} \times(2 \mathrm{~N}+1) \times \mathrm{M}$. N represents the number of components of an input vector. $\mathrm{M}$ is the dimension of the output vector. The number of neurons in the hidden layer can be determined by other methods. In the BP network model, the Tansig function is used as the excitation function of the hidden layer. The excitation function of output layer uses logsig function. The designing step in detail is shown as following.

(1).Number of neurons in the input layer: It is determined by the number of feature extracted from students' experiments. In this paper, five features are extracted.

(2).Number of neurons in the output layer: It is determined by the number of output value. This paper chooses one feature which is a number one to five, so the number of neurons in the output layer is one.

(3).Number of neurons in the hidden layer: There are many ways to determine the number of hidden neurons, including pruning method, complexity adjustment method, gain method, evolution method, adaptive method, etc. Here, use empirical formula which is $H L_{-} n=\sqrt{I_{-} n \times\left(O_{-} n+4\right)}+a$. $a \in[1,10]$. In this formula, $H L_{-} n$ is the number of neurons in the hidden layer, and $I_{-} n$ is the number of neurons in the input layer, and $\mathrm{O}_{-} n$ is the number of neurons in the output layer. In this paper, the number of neurons in the hidden layer is eight.

(4).The activation function: The activation function must be differentiable. S (sigmoid) function is used here. It is as following.

$$
f(x)=\frac{1}{1+e^{-x}}
$$

(5). BP network learning methods: In practice, the BP network is prone to problems that converge to local minimum. This paper chooses the steepest descent BP algorithm. It is along the steepest gradient to modify the weight. The convergence rate is faster than the traditional gradient descent method. This method can reduce the probability of falling into local minimum. 
The structure of the network designed according to the steps is as Fig 1. Using known data, the network is trained. The error of the network is convergent. The error curve of network training is as Fig 2. From Fig 2, it can be seen that the BP network can be used for the actual analysis.

At work, the trained network is used to judge the students' performance in experiments. It gets good effects. In practice, the input vectors maybe include more features of students' performance, so the network can be improved at work.

\section{Five parameters}

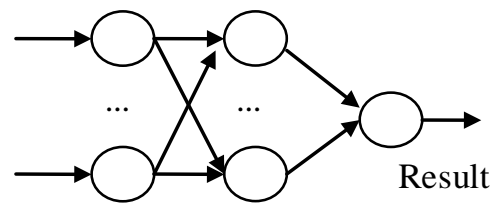

Fig. 1. Structure of BP neural network

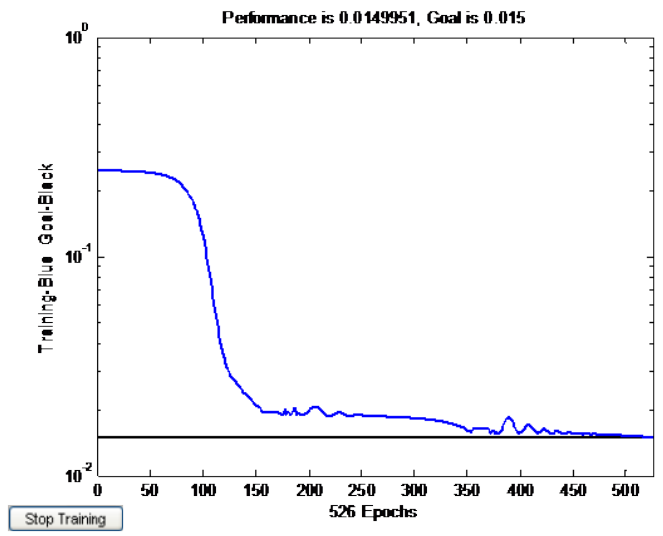

Fig. 2. the training error curve

\section{CONCLUSION}

Objective and correct evaluation of students' experimental performance has always been a key and difficult point in experimental teaching. This paper uses BP neural network to correctly evaluate the experimental performance of students. This method combines the evaluation parameters closely, which is more in line with the actual situation. And it can evaluate students' experimental performance more comprehensively. It merges together students' many features to evaluate students, which is different from traditional evaluation method. This is the main innovation of the paper, too.

\section{ACKNOWLEDGMENT}

This work was supported by the Project: Project of Jiangxi University of Traditional Chinese Medicine (No.2013ZR0074) and Teaching Reform Project of Jiangxi University of Traditional Chinese Medicine (No.2016jzgy-01). And Traditional Chinese Medicine Scientific Research Program of Health Department of Jiangxi Province (No.2013A060).

\section{REFERENCES}

[1] Rakowski R T. Assessment of student performance during industrial training placements[J]. International Journal of Technology and Design Education, 1990(2):106-110.

[2] Linn R L,Burton E. Performance-based assessment: Implications of task specificity[J]. Educational Measurement: Issues and Practice, 1994(13): 5-8,15.

[3] Long Xunjian, Qian Ju, Liang Chuan. Water demand forecast model of BP neutral networks based on principle component analysis. Journal of Chengdu University of Technology(Science \& Technology Edition) Vol.37, No.2, pp206-210, 2010.

[4] Ning Yingying, Li Wenju, Wang Xinnian. Method for vehicle-logo recognition based on principal components analysis and BP neural network. Journal of Liaoning Normal University (Natural Science Edition). Vol.33, No.2, pp179-184, 2010.

[5] Zheng Lili, Li Xiaoqiang, Li Fufeng. Automatic Classification of Lip Color Based on SVM in Traditional Chinese Medicine Inspection. Journal of Biomedical Engineering, Vol.28,No.1,pp7-11, 2011.

[6] Hornik K, Stinchcombe M, White H. Universal approximation of an unknown mapping and its derivatives using multilayer feedforward networks .Neural Networks, 1990, 3 (5) :551-560

[7] YANG Yong, XIE Gangsheng. Handwritten Dig it Recognition Based on BP Neural Network[J]. Journal of East Ch ina Geological Institute, China, 2003, 26 (4): 383-386

[8] TANG Donghong, SUN Houfang, WANG Hongyan. Milling Deformation Forecast with BP Neural Network[J]. Manufacturing Technology \& Machine Tool, China, 2007, 8:48-50

[9] WU Jun, LI Yang. Intrusion detection model based on BP neural network and feature selection[J]. Computer Engineering and Applications, China, 2008,44 (30):114-118. 